\title{
Effet Hall dans des solutions électrolytiques de concentration variable
}

\author{
M. Abbes, R. Gérard, P. Gérard, M. Meton et E. J. Picard \\ Laboratoire de Recherche sur l'Effet Hall, Université Pierre-et-Marie-Curie, U.E.R. Application de la Physique, \\ Tour 46, 4, place Jussieu, 75230 Paris Cedex 05, France
}

(Reçu le 6 juin 1980, révisé le 29 septembre, accepté le 9 octobre 1980)

Résumé. - Nous présentons dans cette lettre les résultats de mesures du nombre de Hall en fonction de la concentration pour trois sels ayant des ions communs. Nous donnons une justification théorique des nombres de Hall ioniques en termes de fonctions d'auto-corrélation des vitesses des ions.

Abstract. - This letter presents the results of measurements of the Hall number versus concentration for three salts having common ions. We propose a theoretical justification of the ionic Hall numbers in terms of time autocorrelation functions of the velocity of ions.

1. Introduction. - Dans une précédente publication [1], nous avons donné un organigramme de l'appareillage expérimental qui a servi aux premières mesures d'effet Hall dans des solutions électrolytiques diluées. Etant donné les importantes modifications apportées au montage, il nous paraît utile de présenter ici un nouvel organigramme et de donner quelques précisions relatives, en particulier, à la nouvelle méthode mise en œuvre pour éliminer la tension de non-alignement. Nous présenterons ensuite les résultats des mesures des nombres de Hall de solutions en fonction de la concentration pour trois électrolytes comportant des ions communs. Ces résultats nous permettent d'obtenir, moyennant certaines hypothèses, les valeurs approchées des nombres de Hall ioniques à dilution infinie.

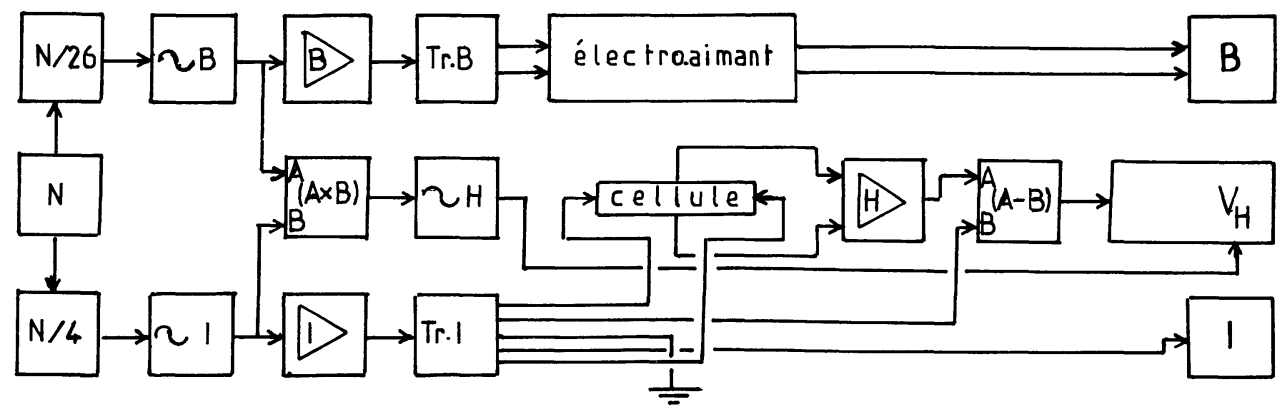

Nouvel organigramme.

[New blockdiagram.]

N : Oscillateur à quartz. Sortie créneaux $1950 \mathrm{~Hz}$.

[Quartz oscillator : square waves $1950 \mathrm{~Hz}$.]

$\mathrm{N} / 26$ et $\mathrm{N} / 4$ : diviseurs de fréquence par 26 et 4.

[Frequency dividers by 26 and 4 .]

$\sim$ B et $\sim$ I : filtres passe-bande centrés sur $75,00 \mathrm{~Hz}$ et $243,75 \mathrm{~Hz}$.

[Band-pass filters.]

$(\mathrm{A} \times \mathrm{B})$ : multiplieur.

[Multiplier.]

B et $\mathrm{D}>$ : amplificateurs de puissance.

[Power amplifiers.]
Tr. B et Tr. I : transformateurs.

[Transformers.]

H) : préamplificateur à faible bruit.

[Low noise preamplifier.]

$(\mathrm{A}-\mathrm{B})$ : soustracteur.

[Subtractor.]

$\mathrm{B}$ et $\mathrm{I}$ : voltmètres numériques.

[Digital voltmeters.]

$\mathrm{V}_{\mathrm{H}}$ : amplificateur sélectif-détecteur synchrone.

[Lock-in amplifier.] 
Nous présenterons enfin, succinctement, une justification théorique des relations liant les nombres de Hall ioniques aux fonctions d'auto-corrélation des vitesses des ions.

2. Aspect expérimental. - On constate sur l'organigramme (1) la présence, à la sortie du préamplificateur d'un filtre réjecteur et d'un filtre passe-bas. Le rôle du réjecteur est d'atténuer la tension de nonalignement $(243,75 \mathrm{~Hz})$, celui du passe-bas, de favoriser la tension de Hall $(168,75 \mathrm{~Hz})$. Ces deux filtres ajoutés à celui de l'amplificateur sélectif du détecteur synchrone présentaient l'inconvénient d'introduire une très grande instabilité qui imposait l'utilisation dans l'étage final du détecteur synchrone d'un filtre passe-bas à très grande constante de temps. Cette méthode d'élimination par filtrage de la tension de non-alignement nous imposait, en outre, de placer dans le circuit de production du courant 2 filtres en série à la sortie du diviseur de fréquence $f / 8$ : un passe-bande et un passe-haut. Sans ce dernier, en effet, nous obtenions une tension à la fréquence de Hall avec le courant seul.

La mise au point d'un soustracteur réalisé à l'aide d'amplificateurs opérationnels du type $148 \mathrm{~A}$ nous a permis d'éliminer les trois filtres mentionnés cidessus : le réjecteur à $243,75 \mathrm{~Hz}$, le passe-bas à $168,75 \mathrm{~Hz}$ et le passe-haut à $342,75 \mathrm{~Hz}$. La tension de non-alignement a pu ainsi être ramenée, dans une première étape, de $10 \mathrm{Vcc}$ environ, à moins de $5 \mathrm{mVcc}$, cette tension résiduelle étant essentiellement à la fréquence de l'harmonique trois du courant. Un nouveau transformateur à sorties symétriques nous a permis de ramener la tension de non-alignement sur l'entrée du détecteur synchrone à moins de $1 \mathrm{mVcc}$ en l'absence de champ magnétique. L'utilisation du soustracteur a entraîné les améliorations suivantes : lors d'une mesure, l'équilibre est atteint très rapidement (gain de plusieurs minutes), les mesures sont plus stables et plus précises, l'écart-type moyen d'une mesure est passé de $25 \mathrm{nV}$ à moins de $10 \mathrm{nV}$. Il nous a permis, enfin, d'effectuer des mesures de tensions de Hall à la fréquence somme $318,75 \mathrm{~Hz}$, aussi bien qu'à la fréquence différence $168,75 \mathrm{~Hz}$.

3. Résultats. - Nous avons disposé pour nos mesures de deux cellules en silice pure d'épaisseurs respectives $d=0,790 \mathrm{~mm}$ et $d=0,200 \mathrm{~mm}$, de largeur $8 \mathrm{~mm}$, de longueur $35 \mathrm{~mm}$. La cellule la plus épaisse nous a permis d'étudier des solutions plus diluées. Nous avons pu faire varier les valeurs efficaces du courant $I_{\text {eff }}$ et du champ magnétique $B_{\text {eff }}$ respectivement de $100 \mu \mathrm{A}$ à $6 \mathrm{~mA}$ et de $0,05 \mathrm{~T}$ à $0,2 \mathrm{~T}$. Pour chaque solution à une concentration $c$ donnée, les mesures ont été faites à la fréquence somme et/ou à la fréquence différence. Les mesures à ces deux fréquences donnent des résultats identiques, si bien que nous ne les avons pas distingués dans les calculs de moyennes.

De la mesure de la tension de Hall $V_{\mathrm{H}}$, nous avons déduit le nombre de Hall :

$$
h=R_{\mathrm{H}} n e=\frac{\sqrt{2} V_{\mathrm{H}} d 9,649 \times 10^{7} c}{I_{\text {eff }} B_{\text {eff }}}
$$

$n$ est la densité des porteurs, $e$ la charge de l'électron, la concentration $c$ est prise en équivalents par $\mathrm{m}^{3}$.

L'ensemble des résultats est donné dans le tableau I, et nous avons représenté figure 1 les valeurs de $h$ en fonction de $100 \sqrt{c}$ ( $c$ étant pris ici en éq. $1^{-1}$ ). Les points figurés par un grand cercle donnent les valeurs moyennes et les écarts moyens obtenus avec la cellule la plus épaisse; les points figurés par un petit cercle donnent les résultats obtenus avec la cellule la plus mince. On peut constater sur ce graphique que les deux cellules donnent des résultats qui se recoupent aux erreurs d'expérience près. Il ne semble donc pas que l'existence d'éventuels mouvements hydrodynamiques conduise à un effet parasité appréciable. On constate, en outre, que les nombres de Hall des

Tableau I. - Valeurs expérimentales des nombres de Hall h en fonction de la concentration pour deux cellules d'épaisseur différente.

[Experimental values of the Hall numbers $h$ versus concentration for two cells of different widths.]

\begin{tabular}{|c|c|c|c|c|c|c|c|c|}
\hline \multicolumn{3}{|c|}{$h_{\mathrm{NaB} \phi_{4}}$} & \multicolumn{3}{|c|}{$h_{\mathrm{NaI}}$} & \multicolumn{3}{|c|}{$h_{\mathrm{NBu}_{4} \mathrm{I}}$} \\
\hline$c$ eq. $\mathrm{m}^{-3}$ & $d=0,79 \mathrm{mn}$ & $d=0,20 \mathrm{~mm}$ & $c$ eq. $\mathrm{m}^{-3}$ & $d=0,79 \mathrm{~mm}$ & $d=0,20 \mathrm{~mm}$ & $c$ eq. $\mathrm{m}^{-3}$ & $d=0,79 \mathrm{~mm}$ & $d=0,20 \mathrm{~mm}$ \\
\hline 2,5 & 0,31 & & 2,5 & $-0,11$ & & 2,5 & $-0,32$ & \\
\hline 3,6 & 0,33 & & 5 & $-0,11$ & $-0,08$ & 3,6 & $-0,33$ & \\
\hline 4,9 & 0,32 & & 10 & $-0,12$ & $-0,11$ & 5 & $-0,30$ & $-0,29$ \\
\hline 6,4 & 0,31 & 0,33 & 20 & & $-0,10$ & 6,4 & $-0,34$ & \\
\hline 8,2 & 0,31 & & 40 & & $-0,12$ & 8,1 & $-0,32$ & $-0,31$ \\
\hline 10 & 0,32 & 0,32 & & & & 10 & $-0,33$ & $-0,30$ \\
\hline 14,4 & & 0,33 & & & & 15 & & $-0,32$ \\
\hline 20 & & 0,35 & & & & 25,6 & & $-0,32$ \\
\hline 25.7 & & 0,34 & & & & & & \\
\hline 40 & & 0,33 & & & & & & \\
\hline
\end{tabular}




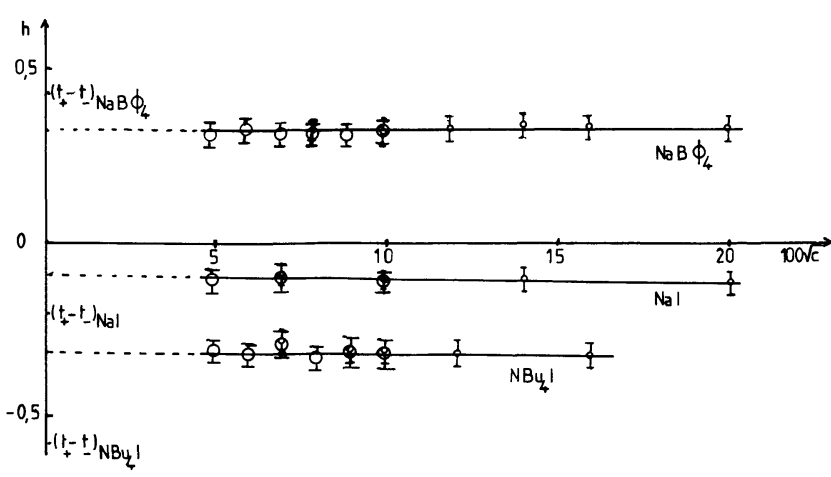

Fig. 1. - Nombres de Hall $h$ en fonction de $100 \sqrt{c} ; c$ concentration en eq. $1^{-1}$. De haut en bas $\mathrm{NaB}_{\phi_{4}}, \mathrm{NaI}, \mathrm{NBu}_{4} \mathrm{I}$. (Les points $\delta$ sont obtenus avec la cellule d'épaisseur $0,79 \mathrm{~mm}$, les points $\Phi_{\text {al }}$ ec la cellule d'épaisseur $0,20 \mathrm{~mm}$.)

[Hall numbers $h$ versus $100 \sqrt{c}: c$ concentration in eq. $1^{-1}$. From top to bottom : $\mathrm{NaB}_{\phi_{4}}, \mathrm{NaI}, \mathrm{NBu}_{4} \mathrm{I}$. (The points ${ }^{\top}$ are obtained with the cell of $0.79 \mathrm{~mm}$ width, the points $\$$ with the cell of $0.20 \mathrm{~mm}$ width).]

solutions étudiées ne varient pas de façon très sensible avec la concentration dans le domaine des concentrations étudié.

4.1 INTER PRÉTATION. - Le nombre de Hall d'une solution d'électrolyte symétrique 1-1 est relié aux nombres de transports et aux nombres de Hall ioniques par la relation [2] :

$$
h=t_{+}^{2} h_{+}-t_{-}^{2} h_{-} .
$$

Les valeurs que nous avons obtenues pour $h$ sont en effet très différentes des nombres $\left(t_{+}-t_{-}\right)$que l'on obtiendrait si les nombres de Hall ioniques étaient égaux à 1 .

Pour les solutions étudiées, les nombres de transports ioniques à dilution infinie sont connus; le graphique de la figure 1 nous donne un ordre de grandeur des nombres de Hall à dilution infinie. En substituant les valeurs obtenues pour chacun des trois sels étudiés dans la relation (2), nous obtenons donc un système de trois équations à 4 inconnues : les 4 nombres de Hall ioniques des deux anions et des deux cations considérés. Si nous faisons l'hypothèse que $h_{\mathrm{B} \phi_{4}} \simeq h_{\mathrm{NBu}_{4}} \simeq 1$, nous trouvons

$$
h_{\mathrm{Na}^{+}}=0,8(0) \quad \text { et } \quad h_{\mathrm{I}^{+}}=0,5(5) \text {. }
$$

Ces valeurs sont sensiblement les mêmes que celles obtenues lors de précédentes mesures d'effet Hall [1,2] portant sur un ensemble plus étendu d'anions et de cations. Ces résultats doivent être encore affinés par de nouvelles expériences plus nombreuses et, si possible, plus précises. Il paraît cependant déjà bien établi que les nombres de Hall ioniques varient de manière importante d'un ion à l'autre, et, en particulier, que les nombres de Hall ioniques des alcalins sont nettement plus grands que ceux des halogènes.
Ces nombres de Hall ioniques sont susceptibles de donner des informations sur la dynamique des ions en solution diluée [6-8], et, plus précisément, sur la fonction d'auto-corrélation des vitesses.

\subsection{FONCTION D'AUTO-CORRÉLATION DES VITESSES} ET THÉORIE DE MORI $\left({ }^{1}\right)$. - La cellule de Hall est un parallélépipède (Fig. 2) au sein duquel un ion, qui constitue une particule lente $(M, q ; \mathbf{R}, \mathbf{P})$ est en interaction avec $N$ molécules de solvant $\left(m, \mathbf{r}_{\alpha} ; \mathbf{p}_{\alpha}\right)$.

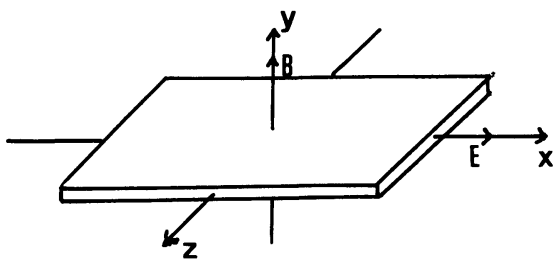

Fig. 2. - Schéma de la cellule de Hall.

[Hall cell geometry.]

La vitesse $\mathbf{V}$ de l'ion est la variable lente du système; elle vérifie, en l'absence de $\mathbf{E}$ et de $\mathbf{B}$ l'équation de Langevin généralisée :

$$
\frac{\mathrm{d}}{\mathrm{d} t} \mathbf{V}(t)=\int_{0}^{t} \mathrm{~d} t^{\prime} \mathbf{V}\left(t-t^{\prime}\right) \tilde{\mathcal{M}}_{\mathbf{v} \mathbf{v}}(t)+\frac{\mathbf{F}(t)}{M}
$$

où $\mathbf{F}(t)$ est la force de Langevin qui varie rapidement.

La fonction de mémoire $\tilde{\mathcal{M}}_{\mathbf{v v}}(t)$ est proportionnelle à la fonction de corrélation de la force de Langevin :

$$
\tilde{\mathcal{M}}_{\mathbf{v v}}(t)=\left(\left\langle V(0)^{2}\right\rangle\right)^{-1}\langle\mathbf{F}(0) \mathbf{F}(t)\rangle .
$$

La fonction de corrélation de la vitesse

$$
\tilde{\mathcal{C}}_{\mathbf{V v}}(t)=\langle\mathbf{V}(0) \mathbf{V}(t)\rangle
$$

évolue de telle façon que

$$
\frac{\mathrm{d}}{\mathrm{d} t} \tilde{\mathfrak{C}}_{\mathbf{v v}}(t)=-\int_{0}^{t} \mathrm{~d} \tau \tilde{\mathcal{C}}_{\mathbf{v v}}(t-\tau) \tilde{\mathscr{M}}_{\mathbf{v v}}(\tau)
$$

et les transformées de Fourier-Laplace $M_{\mathbf{v v}}(\omega)$ et $C_{\mathbf{v v}}(\omega)$ de $\widetilde{\mathcal{H}}_{\mathbf{v v}}(t)$ et $\widetilde{\mathrm{C}}_{\mathbf{v v}}(t)$ sont reliées par l'équation :

$$
C_{\mathbf{v v}}(\omega)=\left\langle V(0)^{2}\right\rangle \frac{1}{-i \omega+M_{\mathbf{v v}}(\omega)} .
$$

Suivant Mori une telle fonction de corrélation peut être développée en une fraction continue [4].

En pratique plusieurs cas peuvent se présenter :

a. Si le porteur est très gros, il peut être assimilé à un Brownon parfait, alors :

$$
C_{V_{x} V_{x}}=\frac{k T}{M} \frac{1}{-i \omega+\Gamma}
$$

( $\left.{ }^{1}\right)$ Les développements qui suivent s'inspirent de la méthode de calcul développée par H. L. Friedman, G. Ciccotti et G. Roepke [3]. 
où $\Gamma=M_{V_{x} V_{x}}(0)$ est, de façon générale, le coefficient de friction statique et où $k T / M=\left\langle V_{x}^{2}\right\rangle$.

(Des considérations de symétrie spatiale, en l'absence de $\mathbf{B}$ et de $\mathbf{E}$ montrent que l'on peut remplacer le problème du mouvement brownien à 3 dimensions par trois problèmes identiques à une dimension.) Dans ce cas particulier le mouvement de l'ion est un processus Markovien.

b. S'il n'en est pas tout à fait ainsi, il peut être suffisant pour obtenir un modèle convenable qui décrive le mouvement de l'ion (processus non Markovien), de tronquer la fraction continue de Mori après une seule itération; on a alors :

$$
C_{V_{x} V_{x}}(\omega)=\frac{k T}{M} \frac{1}{-i \omega+\frac{\omega_{\infty}^{2}}{-i \omega+\frac{\omega_{\infty}^{2}}{\Gamma}}}
$$

où $\omega_{\infty}^{2}=\frac{\left\langle\dot{V}_{x}\right\rangle}{\frac{k T}{M}}$.

c. Il peut être nécessaire d'introduire encore un paramètre supplémentaire $\omega_{\infty}^{\prime 2}$ et de tronquer la fraction continue après deux itérations; alors :

$$
C_{V_{x} V_{x}}(\omega)=\frac{k T}{M} \frac{1}{-i \omega+\frac{\omega_{\infty}^{2}}{-i \omega+\frac{\omega_{\infty}^{\prime 2}}{-i \omega+\frac{\omega_{\infty}^{\prime 2}}{\omega_{\infty}^{2}}} \Gamma}}
$$

où $\omega_{\infty}^{\prime 2}=\frac{\left\langle\ddot{V}_{x}^{2}\right\rangle}{\left\langle\dot{V}_{x}^{2}\right\rangle}-\omega_{\infty}^{2} \ldots$ et ainsi de suite...

4.3 FonCtion DE CORRÉLATION DES VITESSES ET EFFET HALL. - Le quadripôle de Hall a deux entrées, le champ $E_{x}$, linduction $B_{y}$, et deux sorties : le courant de commande lié à $\left\langle V_{x}\right\rangle$ et la tension de Hall liée à $\left\langle V_{z}\right\rangle$.

En présence de $B_{y}$ et de $E_{x}$ on a l'Hamiltonien $H=H_{0}+H_{B}+H_{E}$ et l'opérateur de Liouville $\hat{L}=\hat{L}_{0}+\hat{L}_{B}+\hat{L}_{E}$. On part d'un état d'équilibre avec $B_{y}$ seul puis on applique $E_{x}$. Les réponses à la perturbation $E_{x}$ effectuée dans ces conditions sont :

$$
\begin{aligned}
& \left\langle V_{x}\right\rangle_{E B \lim }=\left\{q \beta \int_{0}^{\infty} \tilde{\mathcal{C}}_{V_{x} V_{x}}^{B} \mathrm{~d} t\right\} E=\left\langle V_{x}\right\rangle_{E \lim } \\
& \left\langle V_{z}\right\rangle_{E B \lim }=\left\{q \beta \int_{0}^{\infty} \tilde{\mathcal{C}}_{V_{x} V_{z}}^{B} \mathrm{~d} t\right\} E=\frac{q \beta}{M}\left\{q \beta \int_{0}^{\infty} t\left\langle V_{x} ; V_{x}(t)\right\rangle_{0} \mathrm{~d} t\right\} E .
\end{aligned}
$$

En effet :

$$
\tilde{\mathcal{C}}_{V_{x} V}^{B}=\int \mathrm{d} \Gamma \exp \left\{-\beta\left(H_{0}+H_{B}\right)\right\} V_{x}(0) \exp \left\{t\left(\hat{L}_{0}+\hat{L}_{B}\right)\right\} V(0) .
$$

Le théorème de Feynman permet d'écrire l'identité

$$
\exp \left\{t\left(\hat{L}_{0}+\hat{L}_{B}\right)\right\}=\exp \left\{t \hat{L}_{0}\right\}+\int_{0}^{t} \mathrm{~d} s \exp \left\{s \hat{L}_{0}\right\} \hat{L}_{B} \exp \left\{(t-s)\left(\hat{L}_{0}+\hat{L}_{B}\right)\right\}
$$

qui donne :

$$
\begin{aligned}
\tilde{\mathrm{C}}_{V_{x} V_{x}}^{B}=\int \mathrm{d} \Gamma \exp \{ & \left.-\beta\left(H_{0}+H_{B}\right)\right\} V_{x}(0) \exp \left\{t \hat{L}_{0}\right\} V_{x}(0)+ \\
& +\int_{0}^{t} \mathrm{~d} s \int \mathrm{d} \Gamma \exp \left\{-\beta\left(H_{0}+H_{B}\right)\right\} V_{x}(0) \exp \left\{s \hat{L}_{0}\right\} \hat{L}_{B} \exp \left\{(t-s)\left(\hat{L}_{0}+\hat{L}_{B}\right)\right\} V_{x}(0)
\end{aligned}
$$

et

$$
\begin{aligned}
\tilde{\mathrm{C}}_{V_{x} V_{z}}^{B}=\int \mathrm{d} \Gamma \exp \{ & \left.-\beta\left(H_{0}+H_{B}\right)\right\} V_{x}(0) \exp \left\{t \hat{L}_{0}\right\} V_{z}(0)+ \\
& +\int_{0}^{t} \mathrm{~d} s \int \mathrm{d} \Gamma \exp \left\{-\beta\left(H_{0}+H_{B}\right)\right\} V_{x}(0) \exp \left\{s \hat{L}_{0}\right\} \hat{L}_{B} \exp \left\{(t-s)\left(\hat{L}_{0}+\hat{L}_{B}\right)\right\} V_{z}(0)
\end{aligned}
$$

ce qui donne, en remplaçant dans ces équations $\hat{L}_{B}$ par $\frac{q B}{M} V_{x} \frac{\partial}{\partial V_{z}}$ et en ne gardant que les termes linéaires en $B$ :

$$
\tilde{\mathcal{C}}_{V_{x} V_{x}}^{B}=\int \mathrm{d} \Gamma \exp \left\{-\beta H_{0}\right\} V_{x}(0) V_{x}(t)+0=\tilde{\mathcal{C}}_{V_{x} V_{x}}^{0}
$$


et

$$
\tilde{\mathrm{C}}_{V_{z} V_{x}}^{B}=0+\frac{q B}{M} \int_{0}^{t} \mathrm{~d} s \int \mathrm{d} \Gamma \exp \left\{-\beta H_{0}\right\} V_{x}(0) V_{x}(t)=\frac{q B}{M}\left(t \tilde{\mathcal{C}}_{V_{x} V_{x}}^{0}\right) .
$$

L'expression de $\hat{L}_{B}$ se calcule à partir de l'Hamiltonien $H_{B}$. Si A est le potentiel vecteur qui décrit l'action de B, on a [5] :

$$
H_{B}=-q \frac{\mathbf{P} \cdot \mathbf{A}}{M}+q^{2} \frac{A^{2}}{2 M} .
$$

Compte tenu de la géométrie de la cellule, nous choisissons $\mathbf{A}=B Z \mathbf{i}$ (i étant le vecteur unitaire suivant $O x$ ). Dans ces conditions :

$$
H_{B}=-\frac{q B}{M} Z\left(P_{x}-q B \frac{Z}{2}\right)
$$

et

$$
\hat{L}_{B}=\frac{q B}{M}\left\{\left(P_{x}-q B Z\right) \frac{\partial}{\partial P_{z}}-Z \frac{\partial}{\partial X}\right\}
$$

L'entrée $B_{y}$ conditionne ou polarise la cellule; elle permet, lorsqu'elle est effectivement branchée, l'observation d'une petite réponse à l'entrée $E_{x}$ sur la sortie $\left\langle V_{z}\right\rangle$.

Dans le cadre de la théorie de la réponse linéaire, nous pouvons poser, en définissant ainsi le nombre de Hall ionique $h_{ \pm}$

$$
\left\langle V_{z}\right\rangle_{E B \lim }=\left\{h_{ \pm} \mu_{\mathrm{e}}^{2} B\right\} E,
$$

$\mu_{\mathrm{e}}$ étant la mobilité électrique. On a donc :

$$
h_{ \pm}=\frac{q}{\mu_{\mathrm{e}}^{2} B} \beta \int_{0}^{\infty} \tilde{\mathcal{C}}_{V_{z} V_{x}}^{B} \mathrm{~d} t
$$

c'est-à-dire

$$
h_{ \pm}=\frac{q^{2}}{\mu_{\mathrm{e}}^{2}} \frac{\beta}{M} \int_{0}^{\infty} t \tilde{\mathrm{C}}_{V_{x} V_{x}}^{0} \mathrm{~d} t
$$

Le nombre de Hall est donc lié à la pente à l'origine de la partie imaginaire de $C_{V_{x} V_{x}}^{0}(\omega)$ :

$$
\begin{aligned}
h_{ \pm}=\frac{q^{2}}{\mu_{\mathrm{e}}^{2} M k T}\left\{\frac{\left.\mathrm{d} J C_{V_{x} V_{x}}^{0}\right\}_{\omega}}{\mathrm{d} \omega}=\right. & = \\
= & \frac{\Gamma^{2}}{\left\langle V_{x}^{2}\right\rangle}\left\{\frac{\mathrm{d} J C_{V_{x} V_{x}}}{\mathrm{~d} \omega}\right\}_{\omega=0}
\end{aligned}
$$

soit en remplaçant $\mu_{\mathrm{e}}^{2}$ par sa valeur

$$
h_{ \pm}=\frac{\int_{0}^{\infty} t \tilde{\mathrm{C}}_{V_{x} V_{x}}^{0} \mathrm{~d} t}{\left\{\int_{0}^{\infty} \mathcal{C}_{V_{x} V_{x}}^{0} \mathrm{~d} t\right\}^{2}}=\frac{\left\{\frac{\mathrm{d}}{\mathrm{d} \omega} J C_{V_{x} V_{x}}^{0}\right\}_{\omega=0}}{\left\{\mathcal{R} C_{V_{x} V_{x}}^{0}\right\}_{\omega=0}^{2}} .
$$

4.4 Effet Hall et théorie de Mori. - Ainsi, dans les différents cas considérés en 4.2 . a $; 4.2$. b et 4.2.c, on a :

a. Pour le brownon parfait $h_{ \pm} \simeq 1$, la seule mesure de $\bar{\mu}_{\mathrm{e}}$ donne la valeur de $\Gamma$.

b. Pour le presque brownon, tel qu'une seule itération soit suffisante $h_{ \pm} \simeq 1-\frac{\Gamma^{2}}{\omega_{\infty}^{2}}$, la mesure de $\mu_{\mathrm{e}}$ donne $\Gamma$; la mesure de $h_{ \pm}$donne alors $\omega_{\infty}^{2}$. Or

$$
\begin{aligned}
\omega_{\infty}^{2}=\frac{1}{M} \frac{\partial^{2} \Sigma U(|X-x|)}{\partial X^{2}}=\frac{n}{3 M} & \times \\
& \times \int \mathrm{d}^{3} \rho g(\rho) \Delta U(\rho)
\end{aligned}
$$

$(\rho=|\mathbf{R}-\mathbf{r}| ; U$ est le potentiel d'interaction ionmolécule de solvant, $\Delta$ l'opérateur Laplacien, $g(\rho)$ la fonction de corrélation spatiale statique, $n$ le nombre d'ions par unité de volume).

$\omega_{\infty}^{2}$ peut donc être calculé si $g(\rho)$ et $U(\rho)$ sont déterminés par d'autres méthodes. La validité de ce modèle du presque brownon peut donc être contrôlée dans ces conditions.

c. Pour le presque brownon correctement décrit par une fonction de corrélation tronquée après deux itérations :

$$
h_{ \pm} \simeq 1-\frac{\Gamma^{2}}{\omega_{\infty}^{2}}+\frac{\omega_{\infty}^{2}}{\omega_{\infty}^{\prime 2}}
$$

la mesure de $\mu_{\mathrm{e}}$ donne $\Gamma$; la mesure de $h_{ \pm}$donne, dans ces conditions une relation entre $\Gamma, \omega_{\infty}^{2}$ et $\omega_{\infty}^{\prime 2}$.

Pour contrôler la validité de ce modèle, il faudrait déterminer par le calcul, non seulement $\omega_{\infty}^{2}$ mais aussi $\omega_{\infty}^{\prime 2}$.

Nos résultats expérimentaux peuvent laisser penser que le modèle a décrit convenablement $\mathrm{NBu}_{4}^{+}$et $\mathrm{B} \phi_{4}^{-}$, que le mođèle $\mathrm{b}$ décrit les halogènes et que le modèle $\mathrm{c}$ s'applique aūx alcalins. 


\section{Bibliographie}

[1] Meton, M., Gérard, P., Picard, E. J., J. Physique Lett. 37 [4] Mori, H., Prog. Theor. Phys. (Japan) 31 (1965) 399. (1976) L-247-L-250.

[2] Meton, M., Thèse Paris (1976). [3] Friedman, H. L., CicCOTTI, G., Roepke, G., à paraître,
Publications du CEAM. Workshop on Stochastic processes in Molecular Dynamics.

[5] Cohen-Tannoudi, C., Diu, B., Laloe, F., Mécanique quantique (Hermann Paris) 1977 (Nouvelle édition), p. 753 et 1486. [6] Friedman, H. L., Ben Naim, A., J. Chem. Phys. 48 (1968) 120

[7] Harris, S., Friedman, H. L., J. Chem. Phys. 50 (1969) 765.

[8] Friedman, H. L., J. Chim. Phys., Numéro spécial, Oct. 1969. 